Supporting Information for

\title{
A Tryptophan Residue Located at the Middle of Putative Transmembrane Domain 11 is Critical for the Function of Organic Anion Transporting Polypeptide 2B1
}

Jialin Bian, Meng Jin, Mei Yue, Meiyu Wang, Hongjian Zhang, and Chunshan Gui ${ }^{*}$

Department of Pharmaceutical Analysis, College of Pharmaceutical Sciences, Soochow University, Suzhou 215123, China

*Correspondence to: Chunshan Gui, Ph.D., 199 Renai Road, Suzhou Industrial Park, Suzhou 215123, China. Phone: +86 512 65882089; Fax: +86 512 65882089; E-mail: guichunshan@suda.edu.cn

\section{SUPPLEMENTARY RESULTS}

The stability of the OATP2B1-E3S complex was investigated by $10-\mathrm{ns}$ molecular dynamics (MD) simulations. As shown in Figure S1A, the whole system is equilibrated after $\sim 5 \mathrm{~ns}$ simulations. The binding of E3S to the binding pocket of OATP2B1 remains stable as the RMSD of E3S is quite small (within $1 \AA$ ) during the whole simulation process (Figure S1B).
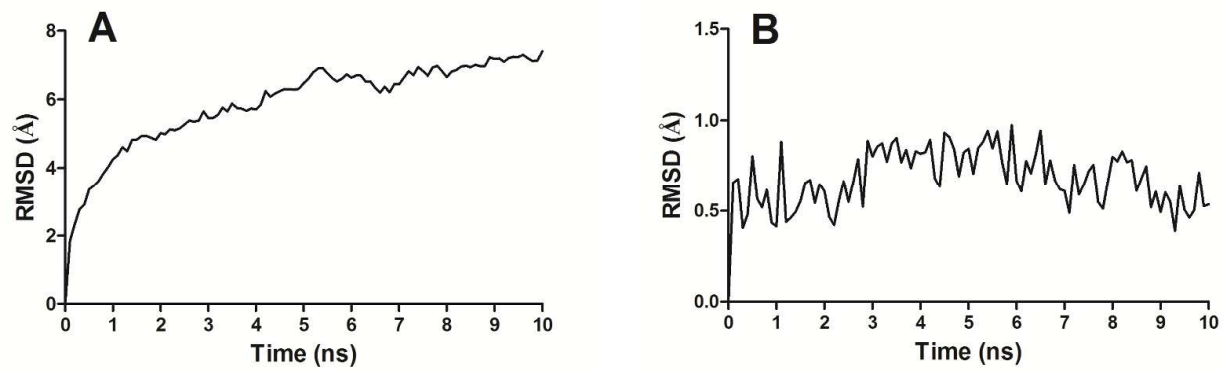

Figure S1. Time evolution of the root mean square deviations (RMSDs) of (A) all $\mathrm{C} \alpha$ atoms of OATP2B1 and (B) all heavy atoms of E3S during the 10-ns MD simulations.

\section{SUPPLEMENTARY EXPERIMENTAL PROCEDURES}

Molecular Dynamics (MD) Simulations. The structure of OATP2B1-E3S complex was embedded in a pre-equilibrated and periodic structure of 1-palmytoyl-2-oleoyl-sn-glycero-3-phosphatidylcholine (POPC) by using the $\mathrm{VMD}^{1}$ program. The lipid molecules within $3 \AA$ of the complex were eliminated. Then the resulting system was inserted into a water box (TIP3 $\mathrm{P}^{2}$ water model) and the waters within $5 \AA$ of the lipid and protein were eliminated. Ten chloride ions were added to neutralize the net positive charge of OATP2B1. The whole system contained OATP2B1, E3S, 278 lipid molecules, 46066 water molecules, and 10 chloride ions for a total of 186367 atoms per periodic cell. The box size was $125 \AA \times 125 \AA \times 153$ Å. 
The system was first equilibrated for $500 \mathrm{ps}$ with the protein fixed. Then, the protein was released and another 500-ps equilibration was performed. Starting from the last frame of the equilibration, we performed 10-ns MD simulations using the $\mathrm{NAMD}^{3}$ package (version 2.92) with the CHARMM ${ }^{4-6}$ force field with explicit water and periodically infinite lipid. In addition, the DREIDING ${ }^{7}$ force field was used to describe the ligand and ligand-protein interactions. Electrostatics were calculated using the particle mesh Ewald (PME) ${ }^{8}$ method with a $12 \AA$ non-bonded cutoff. The constant temperature of $310 \mathrm{~K}$ and the constant pressure of 1 atm were applied by a langevin thermostat and a langevin barostat, respectively. The time step of MD simulations was set to be 1 fs. Data were saved every 1 ps for analysis. Trajectory analyses were carried out with $\mathrm{VMD}^{1}$.

\section{REFERENCES}

(1) Humphrey, W.; Dalke, A.; Schulten, K. VMD: visual molecular dynamics. J. Mol. Graph. 1996, 14, 33-38, 27-38.

(2) Jorgensen, W. L.; Chandrasekhar, J.; Madura, J. D.; Impey, R. W.; Klein, M. L. Comparison of simple potential functions for simulating liquid water. J. Chem. Phys. 1983, 79, 926-935.

(3) Phillips, J. C.; Braun, R.; Wang, W.; Gumbart, J.; Tajkhorshid, E.; Villa, E.; Chipot, C.; Skeel, R. D.; Kale, L.; Schulten, K. Scalable molecular dynamics with NAMD. J. Comput. Chem. 2005, 26, 1781-1802.

(4) Brooks, B. R.; Bruccoleri, R. E.; Olafson., B. D. CHARMM: A program for macromolecular energy, minimization, and dynamics calculations. J. Comput. Chem. 1983, 4, 187-217.

(5) MacKerell, A. D.; Bashford, D.; Bellott, M.; Dunbrack, R. L.; Evanseck, J. D.; Field, M. J.; Fischer, S.; Gao, J.; Guo, H.; Ha, S.; Joseph-McCarthy, D.; Kuchnir, L.; Kuczera, K.; Lau, F. T.; Mattos, C.; Michnick, S.; Ngo, T.; Nguyen, D. T.; Prodhom, B.; Reiher, W. E.; Roux, B.; Schlenkrich, M.; Smith, J. C.; Stote, R.; Straub, J.; Watanabe, M.; Wiorkiewicz-Kuczera, J.; Yin, D.; Karplus, M. All-atom empirical potential for molecular modeling and dynamics studies of proteins. J. Phys. Chem. B 1998, 102, 3586-3616.

(6) Feller, S. E.; MacKerell, A. D. An improved empirical potential energy function for molecular simulations of phospholipids. J. Phys. Chem. B 2000, 104, 7510-7515.

(7) Mayo, S. L.; Olafson, B. D.; Goddard, W. A. DREIDING: A Generic Force Field for Molecular Simulations. J. Phys. Chem. 1990, 94, 8897-8909.

(8) Essmann, U.; Perera, L.; Berkowitz, M. L.; Darden, T.; Lee, H.; Pedersen., L. G. A smooth particle mesh Ewald method. J. Chem. Phys. 1995, 103, 8577-8593. 